\title{
Residual $\beta$ cell function in long-term type 1 diabetes associates with reduced incidence of hypoglycemia
}

\author{
Rose A. Gubitosi-Klug, ${ }^{1}$ Barbara H. Braffett, ${ }^{2}$ Susan Hitt, ${ }^{3}$ Valerie Arends, ${ }^{4}$ Diane Uschner, ${ }^{2}$ Kimberly Jones,,${ }^{5}$ Lisa Diminick, ${ }^{2}$ \\ Amy B. Karger, ${ }^{4}$ Andrew D. Paterson, ${ }^{6,7}$ Delnaz Roshandel, ${ }^{6}$ Santica Marcovina, ${ }^{8}$ John M. Lachin, ${ }^{2}$ Michael Steffes,${ }^{4}$ \\ Jerry P. Palmer, ${ }^{8}$ and the DCCT/EDIC Research Group ${ }^{9}$

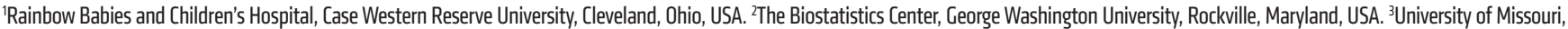 \\ Columbia, Missouri, USA. ${ }^{4}$ University of Minnesota, Minneapolis, Minnesota, USA. ${ }^{~}$ Henry Ford Health System, Detroit, Michigan, USA. ${ }^{6}$ Cenetics and Genome Biology Program, The Hospital for Sick Children, \\ Toronto, Ontario, Canada. 'Divisions of Epidemiology and Biostatistics, Dalla Lana School of Public Health, University of Toronto, Toronto, Ontario, Canada. ${ }^{8}$ University of Washington, Seattle, Washington, \\ USA. ${ }^{9}$ The DCCT/EDIC Research Group is detailed in Supplemental Acknowledgments.
}

BACKCROUND. We investigated residual $\beta$ cell function in Diabetes Control and Complications Trial/Epidemiology of Diabetes Interventions and Complications (DCCT/EDIC) study participants with an average 35-year duration of type 1 diabetes mellitus (T1DM).

\begin{abstract}
METHODS. Serum C-peptide was measured during a 4-hour mixed-meal tolerance test. Associations with metabolic outcomes and complications were explored among nonresponders (all C-peptide values after meal $<0.003 \mathrm{nmol} / \mathrm{L}$ ) and 3 categories of responders, classified by peak C-peptide concentration ( $\mathrm{nmol} / \mathrm{L}$ ) as high $(>0.2)$, intermediate $(>0.03$ to $\leq 0.2)$, and low ( $\geq$ 0.003 to $\leq 0.03$ ).
\end{abstract}

RESULTS. Of the 944 participants, 117 (12.4\%) were classified as responders. Residual C-peptide concentrations were associated with higher DCCT baseline concentrations of stimulated C-peptide ( $P$ value for trend $=0.0001$ ). Residual C-peptide secretion was not associated with current or mean HbA1c, HLA high-risk haplotypes for T1DM, or the current presence of T1DM autoantibodies. The proportion of subjects with a history of severe hypoglycemia was lower with high (27\%) and intermediate $(48 \%)$ residual C-peptide concentrations than with low $(74 \%)$ and no $(70 \%)$ residual C-peptide concentrations ( $P$ value for trend $=\mathbf{0 . 0 0 0 1 )}$. Responders and nonresponders demonstrated similar rates of advanced microvascular complications.

CONCLUSION. $\beta$ Cell function can persist in long-duration T1DM. With a peak C-peptide concentration of $>0.03 \mathrm{nmol} / \mathrm{L}$, we observed clinically meaningful reductions in the prevalence of severe hypoglycemia.

TRIAL REGISTRATION. ClinicalTrials.gov NCT00360815 and NCT00360893.

FUNDING. Division of Diabetes Endocrinology and Metabolic Diseases of the National Institute of Diabetes and Digestive and Kidney Diseases (DP3-DK104438, U01 DK094176, and U01 DK094157).

\section{Introduction}

Insulin secretion declines in most individuals with type 1 diabetes mellitus (T1DM) as duration of diabetes increases, yet residual insulin secretion, as detected by circulating C-peptide after a meal stimulus, has been shown in individuals with long-duration diabetes (1-3). Specifically, type 1 diabetes cohorts with diabetes duration of 30-50 years have demonstrated stimulated C-peptide responses in 10\%-30\% of participants (1-3). The Diabetes Control and Complications Trial (DCCT), in which participants had a T1DM duration of about 10 years, previously demonstrated that

Conflict of interest: ABK declares research support from Siemens Healthcare Diagnostics. DR declares that her spouse is employed by Roche. Copyright: (5) 2021, American Society for Clinical Investigation.

Submitted: August 4, 2020; Accepted: December 9, 2020; Published: February 1, 2021. Reference information: / Clin Invest. 2021;131(3):e143011.

https://doi.org/10.1172/JCl143011. participants from the original intensive group who retained the ability to secrete C-peptide after a mixed-meal stimulus (mixedmeal tolerance test [MMTT]) had significantly lower HbA1c levels, with a $65 \%$ lower risk for severe hypoglycemia, and a $50 \%$ reduced risk for retinopathy progression, compared with participants without a rise in circulating C-peptide after stimulus (4). Since the end of the DCCT, there have been improvements in the analytical sensitivity of C-peptide assays, allowing detection of 10-fold lower concentrations of C-peptide. Our current methodology allows detection of C-peptide levels as low as $0.003 \mathrm{nmol} / \mathrm{L}$ (1). Whether such very low levels of insulin and C-peptide convey beneficial effects over time in T1DM is not fully understood.

Using the more sensitive current assay, we initially explored residual $\beta$ cell function in a pilot study of 58 DCCT participants enrolled in the follow-up Epidemiology of Diabetes Interventions and Complications (EDIC) study with a mean diabetes duration of 25 years. Participants were preselected as likely to 
have maintained residual $\beta$ cell function based on stimulated C-peptide concentrations of $\geq 0.2$ and $\leq 0.5 \mathrm{nmol} / \mathrm{L}$ (i.e., 0.5 $\mathrm{nmol} / \mathrm{L}$ was the highest level permitted for DCCT eligibility) at entry into the DCCT and/or a low mean HbA1c value $(<43$ $\mathrm{mmol} / \mathrm{mol}, 6.1 \%$ ) throughout the DCCT/EDIC study (1). In the pilot study, all 58 participants had detectable concentrations of C-peptide, and $17.2 \%$ retained the ability to secrete C-peptide at concentrations of $>0.03 \mathrm{nmol} / \mathrm{L}$ (1). This cutoff was based on an earlier DCCT study that demonstrated the risk of microvascular disease progression was markedly higher among subjects who entered the trial with C-peptide concentrations of $\leq 0.03$ $\mathrm{nmol} / \mathrm{L}$, reflecting both DCCT entry criteria and the analytical sensitivity of the prior assay (4). While the pilot study confirmed that stimulated C-peptide responses can be detected in DCCT/ EDIC participants with long-duration diabetes, the clinical impact of residual insulin secretion, especially at the lower concentrations detectable with more sensitive current assays, on metabolic control and diabetes complications in long-duration T1DM remained to be explored.

The DCCT/EDIC T1DM cohort, now with an average diabetes duration of 35 years, provided the opportunity to utilize a modern, more sensitive C-peptide assay to evaluate the prevalence of residual $\beta$ cell function in subjects with long-duration diabetes and to characterize individuals with persistent $\mathrm{C}$-peptide secretion. We evaluated C-peptide concentrations, measured at 9 time points during a 4-hour MMTT, in the DCCT/EDIC cohort and classified participants as nonresponders (with all C-peptide values of $<0.003 \mathrm{nmol} / \mathrm{L}$, the lower limit of detection for this assay) versus 3 categories of responders based on the peak C-peptide concentration after stimulus as high $(>0.2 \mathrm{nmol} / \mathrm{L})$, intermediate $(>0.03$ to $\leq 0.2 \mathrm{nmol} / \mathrm{L}$ ), and low ( $\geq 0.003$ to $\leq 0.03 \mathrm{nmol} / \mathrm{L})$. In addition, we assessed the potential beneficial clinical effects of the residual C-peptide response on metabolic outcomes, history of severe hypoglycemia, and microvascular complications.

\section{Results}

As shown in Table 1, the C-peptide study was carried out between 2015 and 2017, when the cohort's mean age ranged between 55.5 and 59.5 years and mean duration of type 1 diabetes was between 34.3 and 36.1 years. Responders were classified based on the peak concentration of their 7 samples after stimulus from the 4-hour MMTT. Seventy-one (7.5\%) of the participants were classified as either high or intermediate responders, demonstrating a stereotypical rise after stimulus in C-peptide secretion (Table 1 and Figure 1). Forty-six (4.9\%) participants were classified as low responders, and 827 (87.6\%) participants were classified as nonresponders (all values below $0.003 \mathrm{nmol} / \mathrm{L}$ ).

High, intermediate, and low responders and nonresponders had similar ages of diabetes onset and duration of diabetes (Table 1 and Supplemental Figure 1; supplemental material available online with this article; https://doi.org/10.1172/ JCI143011DS1). Responders and nonresponders were equally distributed among the intensive and conventional treatment groups as well as among the primary prevention and secondary intervention cohorts. Across C-peptide secretion categories, higher levels were significantly associated with lower HbA1c and insulin requirements at DCCT baseline/eligibility (Table 1).
Higher DCCT baseline 90-minute stimulated C-peptide concentrations were associated with higher EDIC C-peptide concentrations. HbA1c throughout DCCT and EDIC did not differ significantly across responder groups and nonresponders (Table 1), and there were no significant trends across responder categories in insulin requirements during the DCCT after baseline. Current, EDIC mean, and DCCT/EDIC updated mean insulin requirements were higher in response categories with increased levels of residual C-peptide ( $P$ values for all trends $<0.02$ ).

$H L A-D R 3 / D R 4-D Q 8$ haplotypes, major risk factors for type 1 diabetes, were available in a subgroup of 867 participants of White, European ancestry (Table 2). HLA-DR3/DR4-DQ8 haplotypes did not vary among responders and nonresponders $(P=0.0823)$. Four SNPs previously reported to be associated with stimulated C-peptide at DCCT baseline were not associated with current EDIC C-peptide responder status (5).

Type 1 diabetes autoantibody status (GAD-65, IA2, ZnT8), measured concurrent with the C-peptide tests in a case/control set of responders and nonresponders $(n=197)$, is shown in Table 3. GAD- 65 was present in $39 \%$ of the participants, IA2 in $26 \%$, ZnT8 in $4 \%$, while $13.2 \%$ of participants had 2 or more positive antibodies. Autoantibody status was not significantly associated with C-peptide responder status during EDIC, and, in combination, the presence of $H L A-D R 3 / D R 4-D Q 8$ haplotype and autoantibody status was not associated with C-peptide responder status during EDIC (Table 3).

There were significantly fewer severe hypoglycemic events requiring assistance during DCCT/EDIC among high and intermediate responders compared with low responders and nonresponders (Table 4, $P$ value for trend $=0.0001$ during the entire DCCT/ EDIC period; Supplemental Table 1, $P$ value for trend $=0.0003$ during DCCT and $=0.0178$ during EDIC). Similarly, compared with nonresponders, combined high and intermediate responders had a significantly lower history of any severe hypoglycemic events (45\% vs. $70 \%$ for high/intermediate vs. nonresponders, respectively) and recurrent severe hypoglycemia denoted as $>5$ events during DCCT/EDIC (15\% vs. 30\% for high/intermediate vs. nonresponders, respectively). After adjustment for EDIC mean $\mathrm{HbA1c}$, high and intermediate responders were still significantly less likely to experience a severe hypoglycemic event compared with nonresponders $(\mathrm{OR}=0.35,95 \%$ CI $0.21-0.57, P<0.0001)$.

The prevalence of any proliferative diabetic retinopathy (PDR) and clinically significant macular edema (CSME) was similar among all categories of responders (Table 4) and ORs comparing responders to the nonresponders were not significantly different than 1.0 in unadjusted or adjusted analyses. Similarly, there were no differences between responders and nonresponders in the prevalence of kidney disease, as defined as any persistent microalbuminuria or any estimated glomerular filtration rate (eGFR) $<60$ $\mathrm{mL} / \mathrm{min} / 1.73 \mathrm{~m}^{2}$ during DCCT/EDIC (Table 4). Current eGFR, as a continuous predictor, was associated with C-peptide response, with high and intermediate responders having slightly lower eGFR values than nonresponders. This difference remained significant after adjustment for EDIC mean HbA1c $(\beta=-5.41$, standard error $[\mathrm{SE}]=2.01, P=0.0072)$. Of note, there were no significant differences in any acute or chronic complication between low responders versus nonresponders. 
Table 1. Characteristics of C-peptide study participants by EDIC stimulated C-peptide response - EDIC C-peptide study (2015-2017)

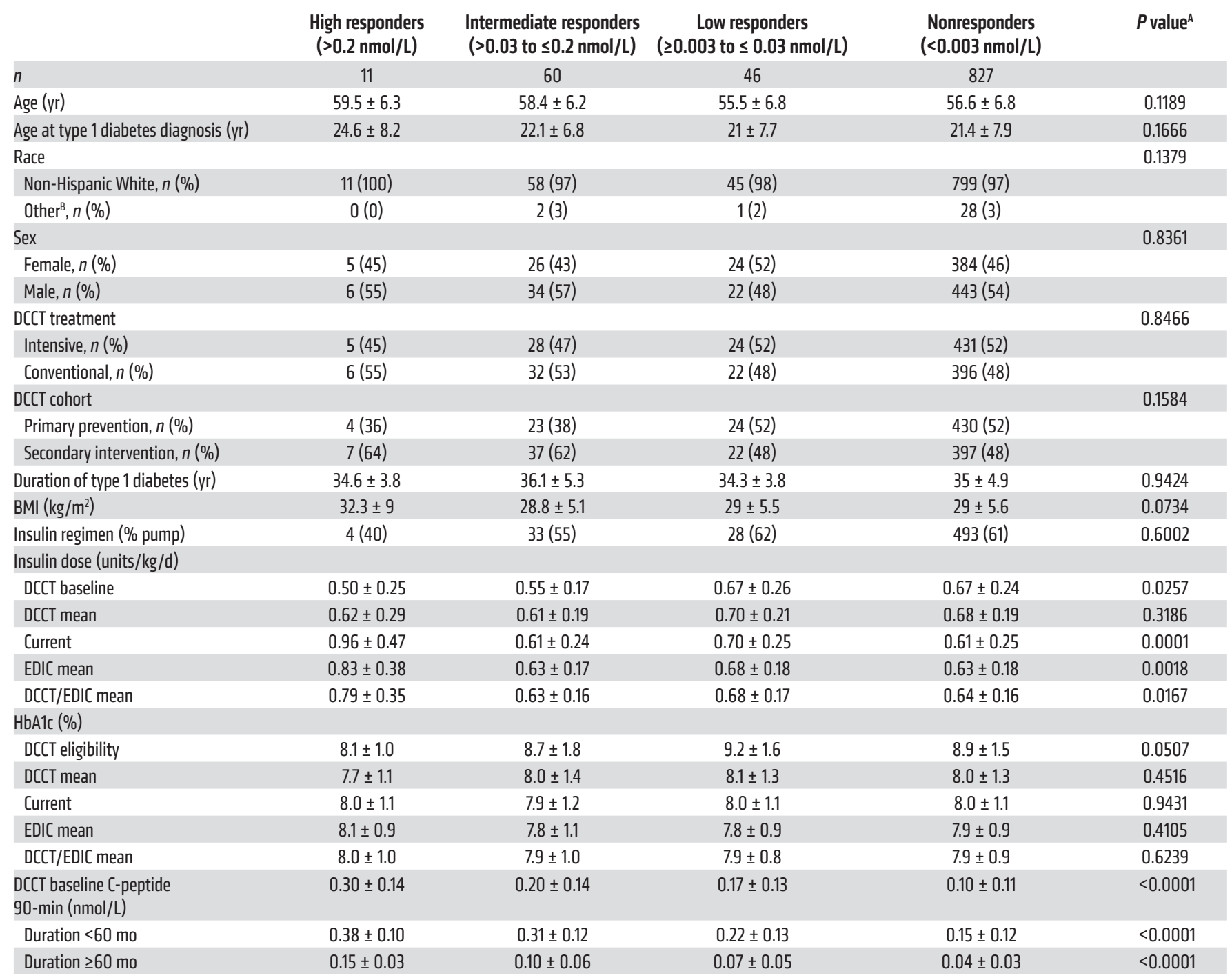

Data are provided as mean \pm standard deviation or $n(\%)$. ${ }^{A}$ The Cochran-Armitage trend test for binary variables or the linear trend test using orthogonal polynomial regression contrasts for continuous variables. ${ }^{B}$ Races indicated by "Other" include non-Hispanic Black, Hispanic, Asian or Pacific Islander, American Indian, and Alaskan Native.

\section{Discussion}

With a diabetes duration averaging 35 years, $12.4 \%$ of the DCCT/ EDIC cohort demonstrated preserved $\beta$ cell function, defined with an analytically sensitive C-peptide assay as a stimulated peak concentration of over $0.003 \mathrm{nmol} / \mathrm{L}$. This long-term preservation of C-peptide secretion, a measure of insulin secretion, was associated with DCCT baseline characteristics of a history of lower HbA1c, higher stimulated C-peptide concentrations, and lower exogenous insulin requirements but not with those metabolic results after baseline. Importantly, concentrations of C-peptide $>0.03 \mathrm{nmol} / \mathrm{L}$ remained significantly associated with lower prevalence of severe hypoglycemia throughout DCCT/EDIC. Concentrations of C-peptide $\leq 0.03 \mathrm{nmol} / \mathrm{L}$ were not associated with long-term benefits related to hypoglycemia, kidney disease, or diabetic retinopathy.
As previously reported, rates of severe hypoglycemia equilibrated between the former intensive and conventional treatment groups, with longer duration of diabetes during EDIC, and persisted at a remarkably high rate of approximately 40 events per 100 patientyears during EDIC follow-up (6). For some participants, the effect of severe hypoglycemia on quality of life was even greater, as approximately one-third of the severe hypoglycemia events occurred in just $7 \%$ of participants, identifying a group of participants that were very susceptible to frequent severe hypoglycemia (6). Our current analyses demonstrate that peak C-peptide concentrations $>0.03 \mathrm{nmo-}$ $1 / \mathrm{L}$ were associated with a $50 \%$ reduced proportion of participants experiencing frequent severe hypoglycemia, from $30 \%$ of nonresponders to $15 \%$ of high and intermediate responders. Thus, one long-term protective factor against the occurrence and reoccurrence of severe hypoglycemia is residual C-peptide secretion. 


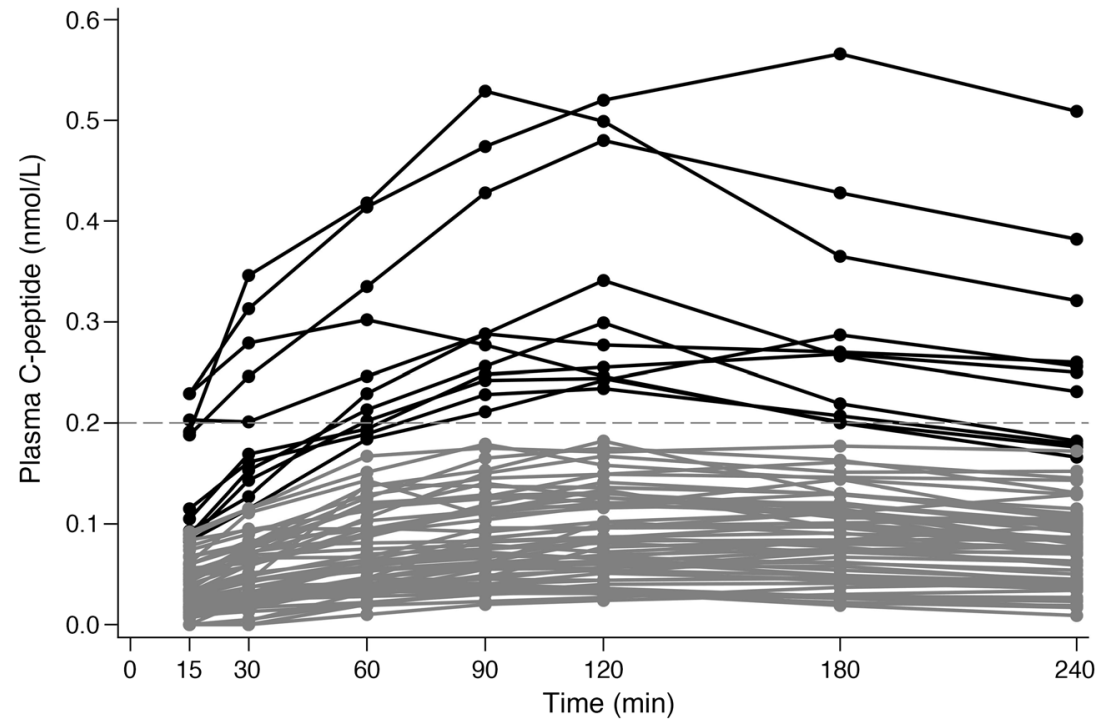

Figure 1. Plasma C-peptide MMTT response curves for high and intermediate responders in EDIC. Plasma C-peptide MMTT response curves in EDIC for high responders $(>0.2 \mathrm{nmol} / \mathrm{L}$, $n=11)$ are shown in black and those for intermediate responders (>0.03 to $\leq 0.2 \mathrm{nmol} / \mathrm{L}, n=60$ ) are shown in gray.

Prior studies have demonstrated that an older age at onset of type 1 diabetes (i.e., the second decade or later in life) is associated with higher residual C-peptide secretion at diagnosis and a slower rate of decline in C-peptide secretion over time (7). The average age of diabetes onset for all responders and nonresponders in our study was above this age threshold, due to the DCCT age and diabetes duration eligibility criteria. Thus, within this group of participants with largely later-onset type 1 diabetes, one might suspect a slower rate of decline and subsequent higher likelihood of residual $\beta$ cell secretion over time; yet our proportion of responders was similar or lower compared with other cohorts with similar diabetes durations $(2,3)$. The variable proportion of responders across cohorts may reflect the underlying heterogeneity of type 1 diabetes and factors modulating $\beta$ cell reserve.

Other studies have investigated the relationship of $\beta$ cell responsiveness in type 1 diabetes at varying diabetes durations to glycemic control and their findings provide additional perspectives on our findings $(8,9)$. Elegant studies of physiology utilizing MMTTs as well as hypoglycemic clamp studies in a smaller cohort of subjects with a shorter duration of diabetes demonstrated that individuals with the most robust counterregulatory hormone responses to hypoglycemia were those with higher concentrations of peak C-peptide on MMTT (8). While we did not directly measure counterregulatory hormone responses, this supports and helps explain the persistent benefit shown in our study against severe hypoglycemia among individuals with greater residual C-peptide secretion. Other measures of $\beta$ cell responsiveness have been used, including assessment of the rate of change or doubling of baseline C-peptide levels during an MMTT (9). Similar to our findings, individuals with long-term type 1 diabetes who retained the ability to respond and double their C-peptide concentration during the MMTT had an older age of onset of diabetes and higher baseline C-peptide concentrations. Notably, the doubling response during MMTT from prior studies utilized a C-peptide assay with a lower limit of detection of $0.02-0.03 \mathrm{nmol} / \mathrm{L}(3,9)$. Interestingly, using the modern, more sensitive immunoassays for C-peptide, of our 117 responders, 67 had a detectable concentration of $\mathrm{C}$-peptide of $\geq 0.003 \mathrm{nmol} / \mathrm{L}$ at time 0 , or baseline, of the MMTT. Sixty-one of the sixty-seven participants (91\%) doubled their starting or time 0 C-peptide concentration at some later time point during the MMTT (i.e., 9 of 11 high, 49 of 53 intermediate, and 3 of 3 low responders; $P$ value for trend $=$ 0.2133). Thus, retaining the ability to double the C-peptide concentration during the MMTT was not significantly different across our high, intermediate, or low responder categories, which are defined based on peak C-peptide concentration. Thus, it is not solely the presence of responsive $\beta$ cells, able to secrete or double even low levels of C-peptide, but the ability to secrete sufficient concentrations of C-peptide that lead to clinically significant benefits.

Understanding the factors - including genetic, immunologic, and metabolic - affecting the $\beta$ cell reserve in type 1 diabetes has been an area of intense research $(4,5,10-12)$. Looking at the potential effect of such factors on long-term residual $\beta$ cell function, $H L A-D R 3 / D R 4-D Q 8$ haplotype did not affect residual $\beta$ cell function in our cohort, similar to what we observed previously with stimulated C-peptide at DCCT baseline (4). Prior GWAS identified a locus on chromosome 1 and 3 loci within the HLA region associated with preservation of C-peptide secretion in DCCT. Of these, 2 loci ( 1 on chromosome 1 and 1 within the HLA region) were independent of the known type 1 diabetes loci (5). However, none of these 4 loci was associated with responder status in this study. Whether these loci, or other novel loci, are involved in $\beta$ cell function with longer duration of type 1 diabetes remains to be explored and may provide further mechanistic insight. Additional investigations, perhaps including the effects of microRNAs as recently reported $(13,14)$, are needed to understand the genetic mechanisms underlying long-term $\beta$ cell function in type 1 diabetes. Additionally, current type 1 diabetes antibody status was not associated with long-term residual $\beta$ cell function. Of note, unlike our earlier evaluations of residual $\beta$ cell function in this cohort (4), current residual $\beta$ cell C-peptide secretion lasting over 3 decades was not influenced by DCCT study randomization to intensive versus conventional therapy. Looking across the DCCT and EDIC study periods, mean HbA1c levels were not significantly different between responders and nonresponders. However, exogenous insulin requirements at DCCT baseline were associated with increased residual C-peptide secretion in EDIC years 22-24. Curiously, this relationship reversed over time, with increased insulin requirements during EDIC. When we adjusted these associations with insulin dose for BMI, the associations were all slightly attenuated, but the $P$ values remain significant (current insulin, $P=$ 0.0006; EDIC mean insulin, $P=0.0060$; DCCT/EDIC updated mean insulin, $P=0.0426$ ). After removing the high responders from the trend testing, comparisons of current, EDIC mean, and 


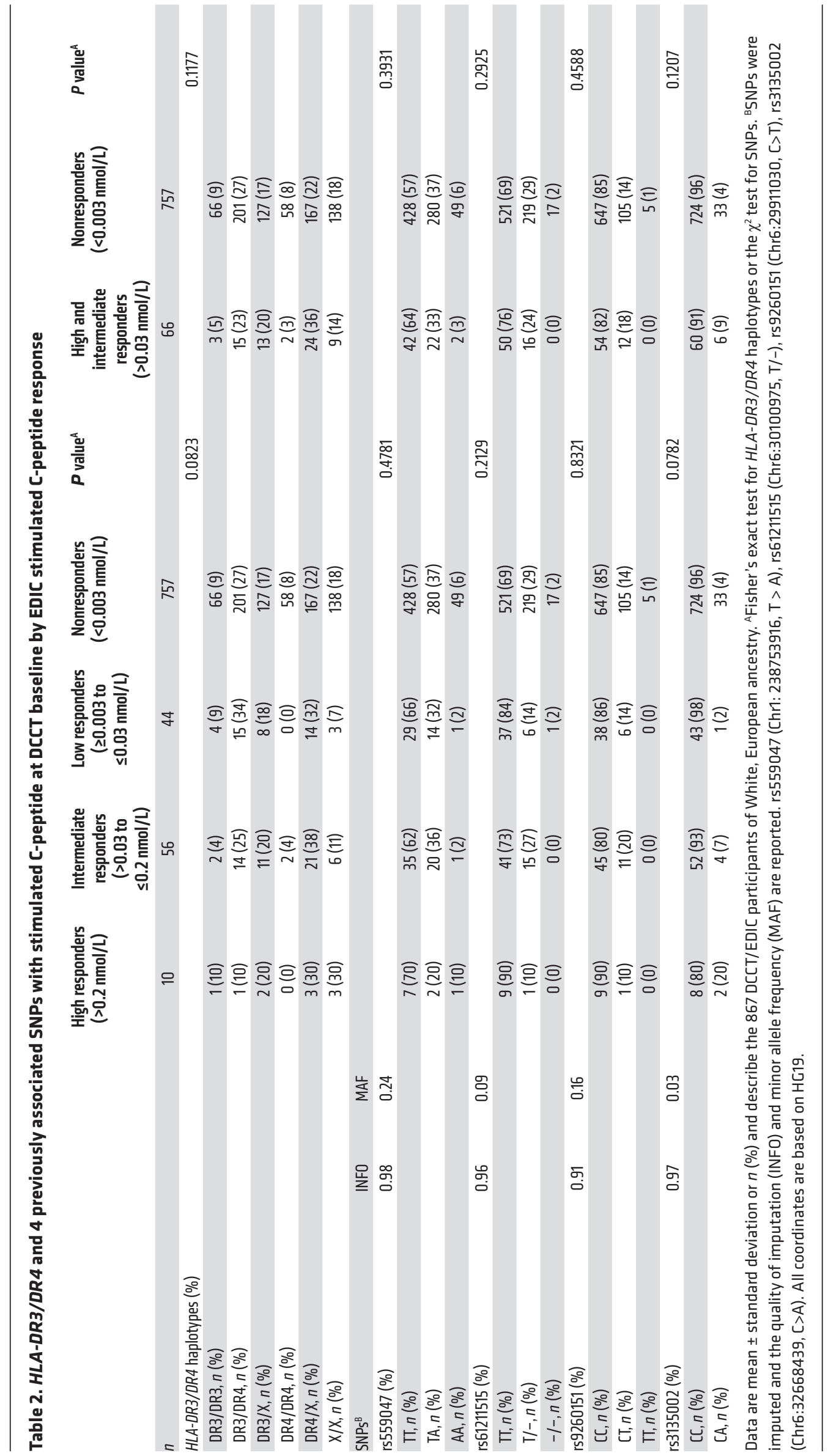


Table 3. Type 1 diabetes autoantibody status at the time of C-peptide collection by EDIC stimulated C-peptide response

\begin{tabular}{|c|c|c|c|c|c|}
\hline & $\begin{array}{l}\text { High responders } \\
(>0.2 \mathrm{nmol} / \mathrm{L}\end{array}$ & $\begin{array}{l}\text { Intermediate responders } \\
\text { (>0.03 to } \leq 0.2 \mathrm{nmol} / \mathrm{L})\end{array}$ & $\begin{array}{c}\text { Low responders } \\
(\geq 0.003 \text { to } \leq 0.03 \mathrm{nmol} / \mathrm{L})\end{array}$ & $\begin{array}{l}\text { Nonresponders } \\
(<0.003 \mathrm{nmol} / \mathrm{L})\end{array}$ & $P$ value \\
\hline$n$ & 11 & 58 & 28 & 100 & \\
\hline \multicolumn{6}{|c|}{ Diabetes autoantibodies (\% positive) ${ }^{A}$} \\
\hline IA2, $n(\%)$ & $3(27)$ & $14(24)$ & $6(21)$ & $29(29)$ & 0.5505 \\
\hline ZnT8, n (\%) & $1(9)$ & $1(2)$ & $2(7)$ & $3(3)$ & 0.7860 \\
\hline$\geq 2$ positive antibodies, $n$ (\%) & $2(18)$ & $8(14)$ & $3(10)$ & $14(14)$ & 0.8937 \\
\hline $\mathrm{HLA}^{+} / \mathrm{AAb}^{+}, n(\%)$ & $6(55)$ & $25(43)$ & $14(50)$ & $41(41)$ & 0.2823 \\
\hline $\mathrm{HLA}^{-} / \mathrm{AAb}^{+}, n(\%)$ & $1(9)$ & $23(40)$ & $11(39)$ & $31(31)$ & \\
\hline $\mathrm{HLA}^{+} / \mathrm{AAb}^{-}, n(\%)$ & $1(9)$ & $2(3)$ & $2(7)$ & $8(8)$ & \\
\hline $\mathrm{HLA}^{-} / \mathrm{AAb}^{-}, n(\%)$ & $2(18)$ & $4(7)$ & $0(0)$ & $11(11)$ & \\
\hline
\end{tabular}

AThe Cochran-Armitage trend test. ${ }^{B}$ Limited to $n=182$ participants with both HLA-DR3/DR4 haplotype and autoantibody status. $P$ value from the Fisher's exact test.

DCCT/EDIC updated mean insulin among intermediate, low, and nonresponders were not significantly different (all trends with $P>$ 0.05). Thus, increased insulin requirements during EDIC observed in the high responders were, in part, mediated by increased insulin requirements due to elevated BMI. Thus, the metabolic effects of hyperglycemia, along with the genetic and immunologic effects on $\beta$ cell reserve, may be complex and vary over the time course of diabetes, affected by age of onset of type 1 diabetes and duration of diabetes as well as by other factors.

Earlier in DCCT and EDIC, residual $\beta$ cell function was associated with lower HbA1c and a reduced rate of a 3-step progression of retinopathy and microalbuminuria. In the current study, with a longer duration of diabetes, progression to mild microvascular disease, including non-PDR and microalbuminuria, was common in our cohort; thus, the development of advanced microvascular events was investigated. The prevalence of microvascular complications among responder and nonresponder categories was similar. Thus, responder status no longer conferred benefit on microvascular disease outcomes in the DCCT/EDIC cohort with a longer duration of diabetes, consistent with the findings of the Pittsburgh Epidemiology of Diabetes Complications Study (15). Considering the central role of glycemia in the etiology of microvascular complications, the absence of an effect of residual $\beta$ cell function, which was not associated with mean HbA1c levels, on advanced microvascular complications is expected. The demonstrated early benefit from responder status may have been tempered or outweighed by diabetes duration and the long-term exposure to hyperglycemia, ultimately leading to continued progression of complications. Other pathophysiologic mechanisms mediating the wellestablished long-term glycemic effects on microvascular disease (e.g., advanced glycation end products) also may play a more prominent role (16). This may, in part, explain the finding that increased long-term residual C-peptide secretion was associated with lower current eGFR, albeit still within the normal range for kidney function, which was significant in both unadjusted and HbA1c-adjusted models. Thus, despite responder levels of C-peptide, the previously described HbA1c-indepen- dent effects of mediators, such as advanced glycation end products, may underlie the slight decline in renal function (16).

The longitudinal study of $\beta$ cell function in type 1 diabetes allows us to speculatively look over time at individual trajectories of peak stimulated C-peptide concentrations. When comparing the DCCT baseline 90-minute responses to the current 90-minute peak MMTT responses for the 11 high responders, response patterns varied over time (Figure 2). Three individuals demonstrated a decline in C-peptide secretion with increasing diabetes duration. Of the remaining 8 participants, 3 individuals demonstrated similar stimulated C-peptide secretion 35 years later; while the remaining 5 individuals appear to have a rise in secretion of C-peptide, despite longer duration of diabetes. Even with the difference in assay methodology, it is striking to note that some individuals secreted at least similar peak stimulated C-peptide concentrations over 3 decades and, in addition, 6 individuals who were nonresponders during the DCCT were identified as responders in the current study (Supplemental Table 2). Future investigations must focus on the mechanisms sustaining $\beta$ cell function, and the potential of endogenously regenerating $\beta$ cells, over the course of type 1 diabetes.

In summary, the ability to maintain $\beta$ cell function by some individuals with type 1 diabetes diagnosed over 35 years ago is associated with factors early in the disease course, including $\beta$ cell function during the first few years after diagnosis and better glycemic control early in the course of type 1 diabetes. In light of our prior investigations documenting the persistent challenge of severe hypoglycemia (6), the salutary effect of residual $\beta$ cell function to reduce the long-term risk of severe hypoglycemia is important, and our longitudinal data suggesting that $\beta$ cell functional decline over time is not inevitable are intriguing. Further study of physiologic mechanisms and pharmacologic strategies to maintain $\beta$ cells and their function holds promise to improve the quality of life for individuals living with type 1 diabetes and should be pursued.

\section{Methods}

The DCCT/EDIC study has been described previously $(17,18)$. Briefly, between 1983 and 1989, 1441 participants with type 1 diabetes, 


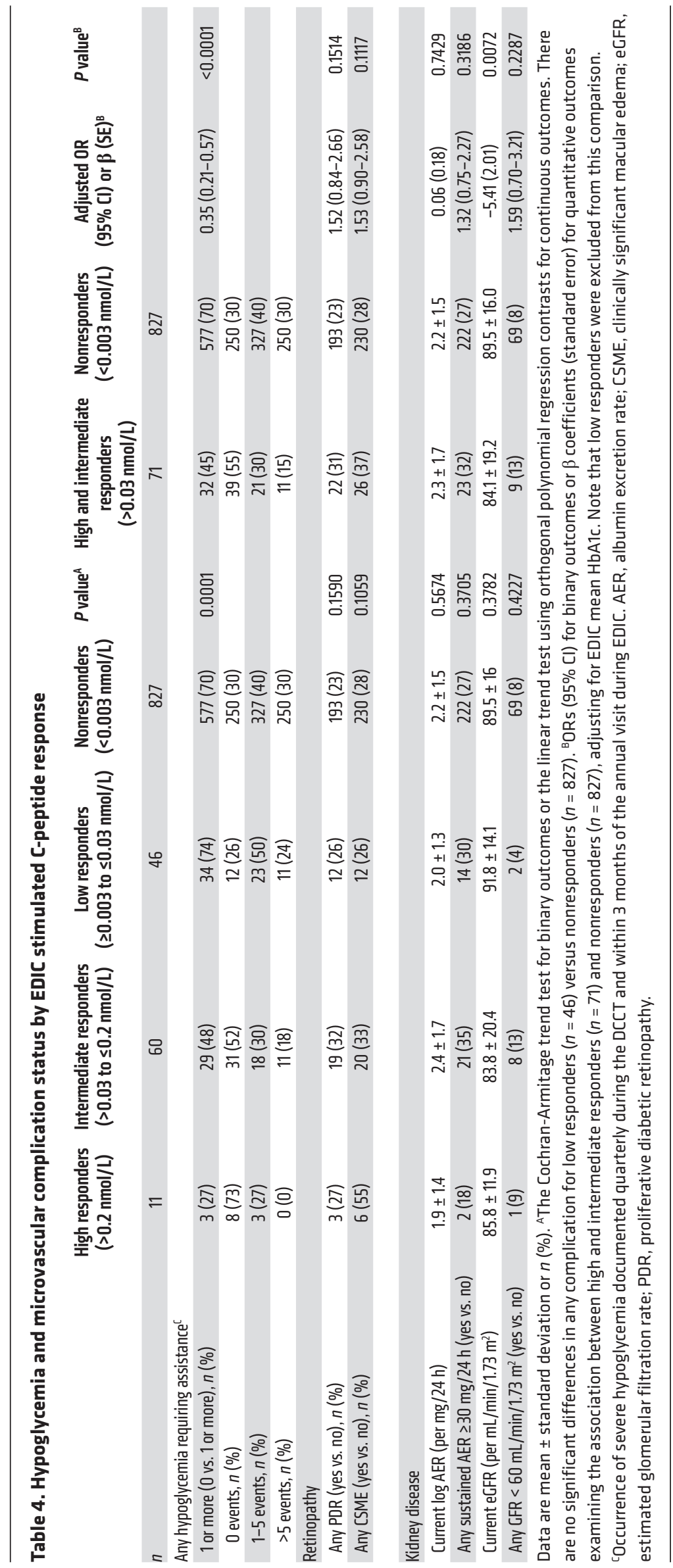

13-39 years of age, were randomized into a multicenter controlled clinical trial designed to compare the effects of intensive therapy, aimed at achieving glycemia as close to the nondiabetic range as safely possible, with conventional diabetes therapy at the time of the study. The DCCT consisted of 2 cohorts: the primary prevention cohort had diabetes for 1-5 years, no retinopathy, urinary albumin excretion $<40 \mathrm{mg}$ per 24 hours, and could have stimulated C-peptide concentrations up to $0.5 \mathrm{nmol} / \mathrm{L} 90$ minutes after a MMTT (4). Candidate participants with basal C-peptide concentrations $\leq 0.2 \mathrm{nmol} / \mathrm{L}$ were eligible for inclusion in the DCCT study (19). The secondary intervention cohort had diabetes for 1-15 years, very-mild-tomoderate nonproliferative retinopathy, and urinary albumin excretion $\leq 200 \mathrm{mg}$ per 24 hours at baseline. Within the secondary cohort, individuals with diabetes duration of 1-5 years had to have stimulated C-peptide $<0.5 \mathrm{nmol} / \mathrm{L}$, whereas those with diabetes duration of 5-15 years had to have stimulated C-peptide $<0.2 \mathrm{nmol} / \mathrm{L}$. Approximately one-half of the sample $(n=711)$ was randomly assigned to intensive therapy ( 3 or more insulin injections daily or subcutaneous infusion with external pump, guided by frequent self-monitoring of blood glucose) with preprandial blood glucose level targets between 70 and $120 \mathrm{mg} / \mathrm{dl}$ (3.9-6.7 mmol/L), a monthly HbA1c target within the nondiabetic range (HbA1c $<43 \mathrm{mmol} / \mathrm{mol}, 6.05 \%$ ), and avoidance of severe hypoglycemia. The remainder $(n=730)$ was assigned to conventional therapy (1-2 daily insulin injections and no numeric blood glucose targets), with the therapeutic goal defined as absence of symptoms of hyperglycemia and avoidance of frequent or severe hypoglycemia.

The treatment groups maintained a separation of median HbA1c levels of about 2 percentage points (7.1\% vs. $9.0 \%$ ) during the mean 6.5 years of DCCT follow-up. Since the DCCT showed that intensive therapy was highly effective in reducing diabetic microvascular complications, intensive therapy was recommended for all participants when the DCCT ended in 1993 (18). Participants were returned to their own health care providers for ongoing diabetes care. In 1994, 1375 (96\%) of the 1428 surviving cohort members volunteered to participate in EDIC for annual observational follow-up (1994 to present). All surviving DCCT/EDIC participants, excluding those with a history of pancreas or islet cell transplant were invited to participate in the EDIC C-peptide study (Figure 3), and 944 participants with type 1 diabetes were enrolled ( $78 \%$ of the surviving cohort). DCCT baseline characteristics between participants $(n=944)$ and nonparticipants $(n=497$; those who refused participation, were loss to follow-up, or were deceased) are provided in Supplemental Table 3. Nonparticipants were less likely to be in the intensive treatment group and had higher insulin dose requirements and higher HbA1c at DCCT baseline than participants. Data collection for this C-peptide study spanned EDIC years 22-24 (2015-2017).

EDIC evaluations. Annual EDIC assessments included a detailed medical history and physical examination with measurements of height, weight, and sitting blood pressure 


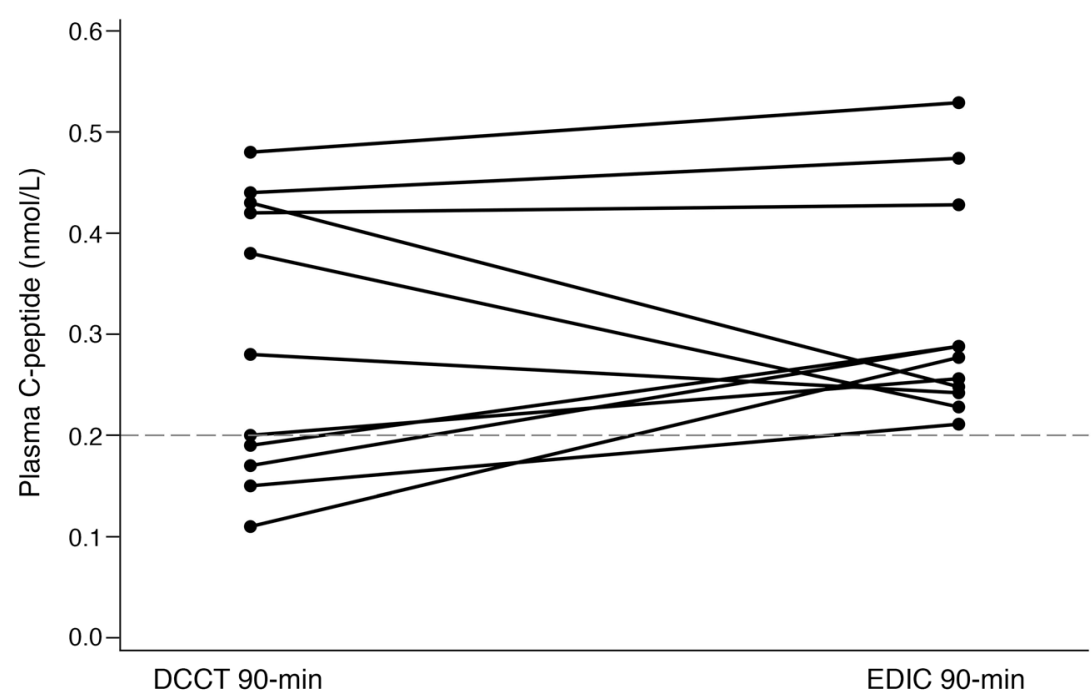

Figure 2. Comparison of DCCT and EDIC plasma peak C-peptide concentrations for high responders $(>0.2 \mathrm{nmol} / \mathrm{L}, n=11)$.

(20). Insulin doses were self-reported and expressed as the average total daily dose in units per kilogram of body weight. Blood samples were assayed centrally for HbA1c using high-performance ion-exchange liquid chromatography (21). Albumin excretion rates (AER) were measured annually during DCCT and on alternate years during EDIC. AER was measured from 4-hour urine samples from DCCT baseline to EDIC year 18 and subsequently from spot urine samples, with AER estimated using the ratio of urine albumin and creatinine concentrations (22). Albumin was initially measured using a fluoroimmunoassay from DCCT baseline to EDIC year 18 and subsequently measured by an immunoassay (Roche). Creatinine in serum and urine was assayed by an enzymatic, IDMS-traceable method (Roche). eGFRs were calculated using the Chronic Kidney Disease Epidemiology Collaboration equation from measured serum creatinine. Kidney disease was defined as an impaired eGFR $\left(<60 \mathrm{~mL} / \mathrm{min} / 1.73 \mathrm{~m}^{2}\right)$ or as persistent microalbuminuria (AER $\geq 30 \mathrm{mg} / 24$ hours on $\geq 2$ consecutive visits) at any point during the DCCT/EDIC study.

Standardized 7-field fundus photographs were obtained every 6 months during DCCT and in a quarter of the cohort annually during EDIC and graded centrally (23). PDR was defined by neovascularization observed on fundus photograph grading or self-reported and/or confirmed scatter photocoagulation at any point during the DCCT/ EDIC study. CSME was defined using fundus photography grading or self-reported and/or confirmed focal photocoagulation or anti-vascular endothelial growth factor therapy at any point during the DCCT/EDIC study. PDR and CSME were the outcomes selected to represent progression to a retinal disease state requiring treatment, given the ubiquitous presence of earlier stages of retinopathy in this long-term cohort. Analyses related to retinopathy were restricted to participants who had the relevant retinopathy assessments within 4 years of the current EDIC C-peptide study ( $n=848$ for PDR, and $n=812$ for CSME).

Severe hypoglycemia was defined as an episode with symptoms or signs consistent with hypoglycemia in which the participant required the assistance of another person. During the DCCT, subjects were asked to report all episodes of suspected severe hypoglycemia immediately, and all subjects were interviewed regarding the episodes using a standard series of questions. During EDIC, the ascertainment of events was restricted to the 3-month window prior to the annual visit and used the same standardized questions.

MMTT. A 4-hour MMTT was administered, and 9 timed plasma specimens were collected and assayed for C-peptide using a chemiluminescent immunoassay (Roche). Participants were asked to ingest a high-carbohydrate diet of at least 150 $\mathrm{g}$ carbohydrate per day for 3 full days followed by a fast of 10-16 hours prior to the MMTT. During the fast, participants refrained from ingesting coffee, tea, caffeine, or alcohol and avoided tobacco and vigorous exercise. The participant remained at rest throughout the procedure. An i.v. line was placed in an antecubital vein and a fingerstick glucose reading taken. Ten minutes after the line was established, the first blood sample was collected (-10 minute time point). Blood was collected into an iced $6 \mathrm{~mL}$ EDTA plasma tube at each time point. Ten minutes later, urine and blood were collected at "baseline" (0 minute time point), after which the participant immediately consumed $6 \mathrm{ml} / \mathrm{kg}$ body weight (maximum $360 \mathrm{ml}$ ) of Boost High Protein Very Vanilla Nutritional Drink over 5 minutes and the clock was started. Blood was collected at after baseline time points 15, $30,60,90,120,180$, and 240 minutes. Plasma tubes were kept on ice before and after blood collection to prevent peptide degradation prior to centrifugation; tubes were centrifuged within 30 minutes of collection. Two urine collections, between 0 and 120 minutes and between 120 and 240 minutes, were collected by pooling, mixing, and decanting the urine into borate-treated tubes. All samples were processed and frozen at the clinical centers and then shipped on dry ice to the EDIC Central Biochemistry Laboratory at the University of Minnesota. All plasma and urine samples were stored at $-70^{\circ} \mathrm{C}$ prior to analysis.

C-peptide was assayed on a Roche Cobas 6000 (Roche Diagnostics) using a sandwich electrochemiluminescent immunoassay traceable to the National Institute for Biological Standards and Control WHO International Reference Reagent for C-peptide (code 84/510). The interassay CVs were $3.5 \%-4.0 \%$ in plasma and $3.0 \%-3.4 \%$ in urine, and the C-peptide assay limit of detection was $0.003 \mathrm{nmol} / \mathrm{L}$. Glucose was measured on a Roche Cobas 6000 using a hexokinase method; interassay CVs were $1.3 \%-1.8 \%$. All time points for a given participant were assayed in a single testing batch.

Responders were defined as participants with any detectable plasma C-peptide concentration after stimulus $\geq 0.003 \mathrm{nmol} / \mathrm{L}$. Responders were subcategorized into 3 groups: high $(>0.2 \mathrm{nmol} / \mathrm{L})$, intermediate $(>0.03$ to $\leq 0.2 \mathrm{nmol} / \mathrm{L}$ ), and low $(\geq 0.003$ to $\leq 0.03$ $\mathrm{nmol} / \mathrm{L}$ ) responders. The low responders were defined as participants with at least 1 of 7 samples after stimulus during the MMTT exhibiting a rise or peak C-peptide as low as the assay limit of detection $(0.003$ $\mathrm{nmol} / \mathrm{L}$ ) and up to $0.03 \mathrm{nmol} / \mathrm{L}$, the latter a concentration chosen based on an earlier DCCT study (24), which demonstrated that the risk of microvascular disease progression was markedly higher among participants who entered the DCCT with C-peptide below this concentration. Intermediate responders were defined as participants with a peak C-peptide in the range of 0.03 up to $0.2 \mathrm{nmol} / \mathrm{L}$, the upper limit based on the previous DCCT findings, which demonstrated significant 


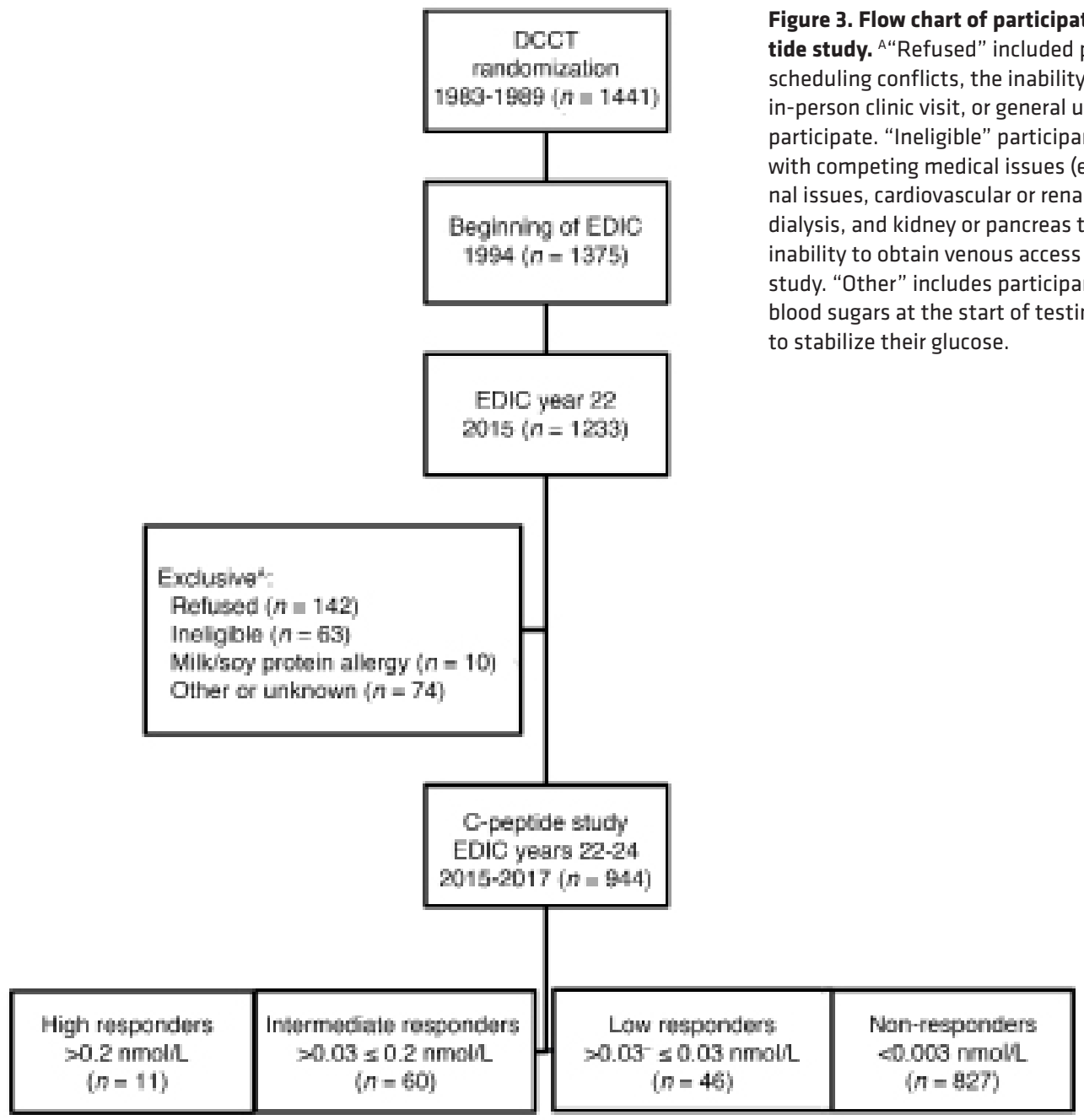

metabolic benefits, including lower fasting blood glucose, glucose rise after stimulus, and lower HbA1c (25). The final group of high responders was defined as participants with peak C-peptide $>0.2 \mathrm{nmol} / \mathrm{L}$.

Results of MMTTs performed at entry into the DCCT ("DCCT baseline") are utilized in analyses. As previously described, the MMTTs during the DCCT were 90-minute studies, with 2 timed specimens collected at time 0 and 90 minutes (4). During the DCCT, C-peptide was measured centrally by radioimmunoassay with the Novo M1230 antibody with a lower limit of detection of $0.03 \mathrm{nmol} / \mathrm{L}$ (4).

Genotyping and HLA imputation. Informed consent was obtained from participants for genetic studies of diabetes and its complications. Whole blood for DNA was collected in 1418 DCCT/EDIC participants toward the end of the DCCT study (1991-1993) and subsequently processed at the EDIC Central Biochemistry Lab at the University of Minnesota. At the Genetics and Genome Biology Program laboratory, DNA samples from all participants were genotyped with the Illumina $1 \mathrm{M}$ BeadArrays (26). Ungenotyped SNPs were imputed using 1000 Genomes data (https://www.internationalgenome.org/; phase 3 , v5). HLA-DR3 and DR4-DQ8 haplotypes, the type 1 diabetes major risk factors, were imputed using 2 SNPs, rs2187668 and rs7454108, as described before (27). They were also imputed using SNP2HLA (http:// software.broadinstitute.org/mpg/snp2hla; ref. 28) and the Type 1 Diabetes Genetic Consortium (https://repository.niddk.nih.gov/studies/ t1dgc/) data set as reference. HLA-DR3 and DR4-DQ8 haplotypes imputed using the 2 methods perfectly matched. Only $n=867$ subjects from European ancestry defined by genetic principle component analysis were included in the genetic association testing (6).

Diabetes autoantibody testing. A sample of 100 responders ( 71 high and intermediate responders and 29 low responders with the next highest detectable C-peptide concentrations $>0.008 \mathrm{nmol} / \mathrm{L}$ ) and 100 randomly selected nonresponders were tested for autoantibody values (IA2, ZnT8) at Northwest Lipid Laboratories. GAD-65 autoantibody was measured in all participants. A fresh, previously unthawed plasma sample was utilized and assayed in batches after study-wide completion of the MMTTs. Testing for values of 3 autoantibodies, GAD-65, IA2, and ZnT8, was performed based on a protocol approved by the National Institute of Diabetes and Digestive and Kidney Diseases (NIDDK) Autoantibody Harmonization Committee (29). Plasma samples were analyzed for antibodies utilizing a radioligand-binding assay as previously described (29). Concentrations of GAD-65 and IA2 autoantibodies were determined from a NIDDK standard curve, and the results are reported as NIDDK units and NIDDK units/mL, respectively. A sample with a GAD- 65 autoantibody result of $\geq 33 \mathrm{DK}$ units $/ \mathrm{mL}$ or an IA2 result $\geq 5 \mathrm{DK}$ units/mL was considered positive for antibodies. The ZnT8 autoantibody assay uses ZnT8-RW dimer cDNA in the in vitro transcription and translation system. The results are expressed as an 
index using counts per minute (CPM) ([sample CPM - negative control $\mathrm{CPM}] /$ [positive control CPM - negative control CPM]). The upper limit of normal (0.020) was established as the 99th percentile from receiver operating characteristic curves in 100 control subjects without diabetes and 50 patients with new-onset type 1 diabetes $(29,30)$.

Statistics. Differences in demographic and clinical characteristics between responders and nonresponders were tested using the Cochran-Armitage trend test for binary variables or the linear trend test using orthogonal polynomial regression contrasts for continuous variables. Insulin dose and $\mathrm{HbA1c}$ were assessed using the baseline value, DCCT mean, current value, EDIC mean, and DCCT/EDIC time-weighted mean. The DCCT and EDIC updated means represent the running averages during the DCCT and EDIC, respectively. The time-weighted DCCT/EDIC mean HbA1c represents the total glycemic exposure during DCCT/EDIC and was calculated by weighting each value by the time interval since the last measurement. Differences between response categories were tested using Fisher's exact test for $H L A-D R 3 / D R 4$ haplotypes or the $\chi^{2}$ test for SNPs. Linear and logistic regression models were used to evaluate the association of history of severe hypoglycemia, retinopathy, and kidney disease with C-peptide response status (high and intermediate vs. nonresponders), adjusting for EDIC mean HbA1c. Given the exploratory nature of our analyses, no adjustments were made for multiplicity, and $P \leq 0.05$ indicates nominal significance.

Study approval. The EDIC C-peptide study was approved by the institutional review boards at all sites (University Hospitals Cleveland Medical Center, Cleveland, Ohio, USA; University of Pennsylvania, Philadelphia, Pennsylvania, USA; Cornell University, Ithaca, New York, USA; Henry Ford Health System; Joslin Diabetes Center, Boston, Massachusetts, USA; Massachusetts General Hospital, Boston, Massachusetts, USA; Mayo Clinic, Rochester, Minnesota, USA; Medical University of South Carolina, Charleston, South Carolina, USA; Health Partners Institute, Minneapolis, Minnesota, USA; University of Iowa, Iowa City, Iowa, USA; University of Minnesota; University of Missouri-Columbia, Columbia, Missouri, USA; University of Pittsburgh, Pittsburgh, Pennsylvania, USA; University of Tennessee Health Science Center, Memphis, Tennessee, USA; University of Texas Southwestern, Dallas, Texas, USA; University of Toronto; University of Washington, Seattle, Washington; University of Western Ontario, London, Ontario, Canada; Vanderbilt University, Nashville, Tennessee, USA; Washington University in St. Louis, St. Louis, Missouri, USA; Yale University, New Haven, Connecticut, USA; Northwestern University, Chicago, Illinois, USA; University of California San Diego, San Diego, California, USA; University of Maryland, Baltimore, Maryland, USA; Uni- versity of New Mexico, Albuquerque, New Mexico, USA; University of South Florida, Tampa, Florida, USA; University of Michigan, Ann Arbor, Michigan, USA), and all participants provided written informed consent for biochemical assessments, complications surveillance, and genetic testing.

\section{Author contributions}

RAGK, BHB, and JPP designed the analyses and wrote the manuscript. SH, VA, MS, ABK, KJ, LD, DU, ADP, DR, SM, and JML wrote portions of the manuscript and reviewed and edited the manuscript. BHB and DU conducted the statistical analyses and prepared the tables and figures.

\section{Acknowledgments}

See Supplemental Acknowledgments for DCCT/EDIC Research Group details. EDIC is funded by a NIH cooperative agreement (U01), a support mechanism that entails substantial involvement from NIH scientific staff. RAGK and BHB are the guarantors of this work and, as such, had full access to all of the data in the study and take responsibility for the integrity of the data and the accuracy of the data analysis. RAGK had final responsibility for the decision to submit for publication. Industry contributors have had no role in the conduct of the DCCT/EDIC study but have provided free or discounted supplies or equipment to support participants' adherence to the study protocols; these contributors include Abbott Diabetes Care, Animas, Bayer Diabetes Care, BD, Eli Lilly, Extend Nutrition, Insulet Corporation, LifeScan, Medtronic Diabetes, Nipro Home Diagnostics, Nova Diabetes Care, Omron, Perrigo Diabetes Care, Roche Diabetes Care, and Sanofi-Aventis. DCCT/ EDIC has been supported by cooperative agreement grants (19821993, 2012-2017, 2017-2022) and contracts (1982-2012) with the Division of Diabetes, Endocrinology, and Metabolic Diseases of the NIDDK (U01 DK094176 and U01 DK094157) as well as by the National Eye Institute, the National Institute of Neurologic Disorders and Stroke, the General Clinical Research Centers Program (1993-2007), and Clinical Translational Science Center Program, Bethesda, Maryland, USA (2006 to present). Additional support for this DCCT/EDIC collaborative study was provided by the NIH through the NIDDK grant 1-DP3-DK104438.

Address correspondence to: Barbara H. Braffett, The Biostatistics Center, George Washington University, 6110 Executive Blvd., Suite 750, Rockville, Maryland 20852, USA. Phone: 301.881.9260; Email: braffett@bsc.gwu.edu.
1. McGee P, et al. Insulin secretion measured by stimulated C-peptide in long-established Type 1 diabetes in the Diabetes Control and Complications Trial (DCCT) / Epidemiology of Diabetes Interventions and Complications (EDIC) cohort: a pilot study. Diabet Med. 2014;31(10):1264-1268.

2. Oram RA, et al. The majority of patients with long-duration type 1 diabetes are insulin microsecretors and have functioning beta cells. Diabetologia. 2014;57(1):187-191.

3. Keenan HA, et al. Residual insulin production and pancreatic $\beta$-cell turnover after 50 years of diabetes: Joslin Medalist Study. Diabetes.
2010;59(11):2846-2853.

4. The DCCT Research Group. Effect of intensive therapy on residual beta-cell function in patients with type 1 diabetes in the diabetes control and complications trial. A randomized, controlled trial. Ann Intern Med. 1998;128(7):517-523.

5 . Roshandel D, et al. Meta-genome-wide association studies identify a locus on chromosome 1 and multiple variants in the $\mathrm{MHC}$ region for serum C-peptide in type 1 diabetes. Diabetologia. 2018;61(5):1098-1111.

6. Gubitosi-Klug RA, et al. Risk of severe hypoglycemia in type 1 diabetes over 30 years of fol- low-up in the DCCT/EDIC study. Diabetes Care. 2017;40(8):1010-1016.

7. Davis AK, et al. Prevalence of detectable C-Peptide according to age at diagnosis and duration of type 1 diabetes. Diabetes Care. 2015;38(3):476-481.

8. Rickels MR, et al. High residual C-peptide likely contributes to glycemic control in type 1 diabetes. J Clin Invest. 2020;130(4):1850-1862.

9. Yu MG, et al. Residual $\beta$ cell function and monogenic variants in long-duration type 1 diabetes patients. J Clin Invest. 2019;129(8):3252-3263.

10. Steck AK, et al. Predicting progression to diabetes in islet autoantibody positive children. JAutoim- 
mun. 2018;90:59-63.

11. Redondo MJ, et al. A type 1 diabetes genetic risk score predicts progression of islet autoimmunity and development of type 1 diabetes in individuals at risk. Diabetes Care. 2018;41(9):1887-1894.

12. McKeigue PM, et al. Persistent C-peptide secretion in Type 1 diabetes and its relationship to the genetic architecture of diabetes. BMC Med. 2019;17(1):165.

13. Samandari N, et al. Circulating microRNA levels predict residual beta cell function and glycaemic control in children with type 1 diabetes mellitus. Diabetologia. 2017;60(2):354-363.

14. Snowhite IV, et al. Association of serum microRNAs with islet autoimmunity, disease progression and metabolic impairment in relatives at risk of type 1 diabetes. Diabetologia. 2017;60(8):1409-1422.

15. Williams KV, et al. Persistent C-peptide levels and microvascular complications in childhood onset type 1 diabetes of long duration. J Diabetes Complications. 2019;33(9):657-661.

16. Genuth S, et al. Skin advanced glycation end products glucosepane and methylglyoxal hydroimidazolone are independently associated with long-term microvascular complication progression of type 1 diabetes. Diabetes. 2015;64(1):266-278.

17. The DCCT Research Group. Diabetes Control and Complications Trial (DCCT): Results of feasibility study. Diabetes Care. 1987;10(1):1-19.
18. The DCCT Research Group. The effect of intensive treatment of diabetes on the development and progression of long-term complications in insulin-dependent diabetes mellitus. $\mathrm{N} \mathrm{Engl} \mathrm{J}$ Med.1993;329(14):977-986.

19. The DCCT Research Group. The Diabetes Control and Complications Trial (DCCT). Design and methodologic considerations for the feasibility phase. Diabetes. 1986;35(5):530-545.

20. The DCCT/EDIC Research Group. Epidemiology of Diabetes Interventions and Complications (EDIC): design, implementation, and preliminary results of a long-term follow-up of the Diabetes Control and Complications Trial Cohort. Diabetes Care. 1999;22(1):99-111.

21. Steffes M, et al. Hemoglobin A1c measurements over nearly two decades: sustaining comparable values throughout the Diabetes Control and Complications Trial and the Epidemiology of Diabetes Interventions and Complications study. Clin Chem. 2005;51(4):753-758.

22. Younes N, et al. Comparison of urinary albumin-creatinine ratio and albumin excretion rate in the Diabetes Control and Complications Trial/Epidemiology of Diabetes Interventions and Complications study. Clin J Am Soc Nephrol. 2010;5(7):1235-1242.

23. Early Treatment Diabetic Retinopathy Study Research Group. Early photocoagulation for diabetic macula retinopathy: ETDRS report number
9. Ophthalmology. 1991;98(5 Suppl):766-785

24. Steffes MW, et al. Beta-cell function and the development of diabetes-related complications in the diabetes control and complications trial. Diabetes Care. 2003;26(3):832-836.

25. The DCCT Research Group. Effects of age, duration and treatment of insulin-dependent diabetes mellitus on residual beta-cell function: observations during eligibility testing for the Diabetes Control and Complications Trial (DCCT). JClin Endocrinol Metab. 1987;65(1):30-36.

26. Paterson AD, et al. A genome-wide association study identifies a novel major locus for glycemic control in type 1 diabetes, as measured by both A1C and glucose. Diabetes. 2010;59(2):539-549.

27. Winkler C, et al. Feature ranking of type 1 diabetes susceptibility genes improves prediction of type 1 diabetes. Diabetologia. 2014;57(12):2521-2529.

28. Jia X, et al. Imputing amino acid polymorphisms in human leukocyte antigens. PLoS One. 2013;8(6):e64683.

29. Bonifacio E, et al. Harmonization of glutamic acid decarboxylase and islet antigen-2 autoantibody assays for national institute of diabetes and digestive and kidney diseases consortia. J Clin Endocrinol Metab. 2010;95(7):3360-3367.

30. Yu L, et al. Zinc transporter-8 autoantibodies improve prediction of type 1 diabetes in relatives positive for the standard biochemical autoantibodies. Diabetes Care. 2012;35(6):1213-1218. 